\title{
Multi-targeted tyrosine kinase inhibitors as third-line regimen in advanced non-small cell lung cancer: a network meta-analysis
}

\author{
Zhonghan Zhang ${ }^{1 \#}$, Yuanyuan Zhao ${ }^{1 \#}$, Feiteng Lu ${ }^{1 \#}$, Xue Hou ${ }^{1}$, Yuxiang $\mathrm{Ma}^{2}$, Fan Luo ${ }^{1}$, Kangmei Zeng", \\ Shen Zhao ${ }^{1}$, Yaxiong Zhang ${ }^{1}$, Ting Zhou ${ }^{1}$, Yunpeng Yang ${ }^{1}$, Wenfeng Fang ${ }^{1}$, Yan Huang ${ }^{1}$, Li Zhang ${ }^{1}$, \\ Hongyun Zhao ${ }^{2}$
}

${ }^{1}$ Department of Medical Oncology, ${ }^{2}$ Department of Clinical Research, Sun Yat-sen University Cancer Center, State Key Laboratory of Oncology in South China, Collaborative Innovation Center for Cancer Medicine, Guangzhou 510060, China

Contributions: (I) Conception and design: Z Zhang, H Zhao, L Zhang; (II) Administrative support: None; (III) Provision of study materials or patients: F Lu, Z Zhang; (IV) Collection and assembly of data: X Hou, K Zeng, T Zhou, Y Yang, W Fang, Y Huang; (V) Data analysis and interpretation: Z Zhang, Y Zhao, F Luo; (VI) Manuscript writing: All authors; (VII) Final approval of manuscript: All authors.

\#These authors contributed equally to this work.

Correspondence to: Hongyun Zhao. Department of Clinical Research, Sun Yat-sen University Cancer Center, 651 Dongfeng Road East, Guangzhou 510060, China. Email: zhaohy@sysucc.org.cn; Li Zhang. Department of Medical Oncology, Sun Yat-sen University Cancer Center, 651 Dongfeng Road East, Guangzhou 510060, China. Email: zhangli6@mail.sysu.edu.cn.

Background: Four multi-targeted tyrosine kinase inhibitors (TKIs) including apatinib, anlotinib, fruquintinib and lenvatinib are currently available as third-line regimen for advanced non-small cell lung cancer (NSCLC) patients who failed at least two lines of systemic therapy. Limited evidence was provided to demonstrate the general efficacy and safety profile of these drugs as third-line treatment approach for NSCLC.

Methods: Eligible literature was searched from electronic database. Data of objective response rate (ORR), disease control rate (DCR), progression-free survival (PFS), overall survival (OS), treatment related adverse event (TRAE), treatment related adverse event grade 3-5 (TRAE3-5), hypertension, proteinuria, handfoot skin reaction (HFSR), elevated ALT/AST, nausea and vomiting, diarrhea were synthetically extracted. Multiple-treatments comparisons (MTCs) based on a Bayesian consistency model integrated the efficacy and toxicity outcomes. Rank probabilities of each regimen were assessed and clustered by the surface under the cumulative ranking curve.

Results: Five phase II/III randomized trials involving 915 advanced NSCLC patients were enrolled. MTCs showed that four multi-targeted TKIs shared equivalent efficacy in terms of outcome measures, of which anlotinib stood out in ORR (OR =39.26; 95\% CI: 2.36-2,748.06), DCR (OR =8.69; 95\% CI: 1.70-50.18) and PFS (HR $=0.27 ; 95 \%$ CI: 0.10-0.78) when compared with placebo plus BSC. No significantly differences were observed among these TKIs and placebo with respect to OS, TRAE and TRAE 3-5. Fruquintinib and lenvatinib may relate to high rate of HFSR while anlotinib may relate to hypertension.

Conclusions: Multi-targeted TKIs (apatinib, anlotinib, fruquintinib and lenvatinib) with acceptable efficacy and safety profile were options for advanced NSCLC in third-line setting.

Keywords: Multi-targeted tyrosine kinase inhibitors (multi-targeted TKIs); non-small cell lung cancer (NSCLC); apatinib; anlotinib; fruquintinib; lenvatinib

Submitted May 19, 2019. Accepted for publication Aug 08, 2019.

doi: $10.21037 /$ atm.2019.08.51

View this article at: http://dx.doi.org/10.21037/atm.2019.08.51 


\section{Introduction}

Lung cancer remains a leading cause of cancer-related death globally, having a 5 -year survival rate of less than $20 \%(1,2)$. Non-small cell lung cancer (NSCLC), accounting for the majority of lung cancer cases (approximately $80-85 \%$ ), is usually allocated to advanced stage at their first diagnosis (3). Moreover, a considerable portion of patients with locally advanced NCSLC will relapse with metastases, causing the lower overall 5 -year survival rate $(4,5)$.

Despite a significant improvement in screening, diagnostics and therapy for NSCLC during the past decades, the prognosis for patients with advanced NSCLC remains poor (6-8). The standard care, according to the NCCN guideline, for these locally advanced or metastatic NSCLC patients is systemic palliative chemotherapy and/ or radiotherapy (9). Platinum-based doublet chemotherapy plus immune checkpoint inhibitors (ICIs) is currently being recommended as the standard first-line treatment option for advanced NSCLC, followed by second-line systemic ICIs monotherapy (nivolumab, pembrolizumab and atezolizumab) or other systemic therapy (docetaxel, pemetrexed or gemcitabine) or target therapy (gefitinib and erlotinib) as the subsequent therapy. The emergence of ICIs for the treatment of malignancy has significantly changed the therapeutic landscape of lung cancer, especially NSCLC. Long-term survival and durable responses were observed in a certain proportion of patients with the 5 -year survival rate increased from $4 \%$ to $16 \%$ for advanced NSCLC patients after treating with immunotherapy (10). These encouraging therapies have improved survival and the quality of life for NSCLC patients, however, most of the patients experience drug-resistance or disease progression after receiving a certain period of time with first- or second-line therapy $(11,12)$ and there is currently no standard therapeutic options beyond second-line, placing the subsequent salvage therapy in a difficult situation.

Tumor angiogenesis have been identified as important therapeutic targets for malignant tumor, which is essential in the process of primary tumor growth, proliferation, differentiation and metastasis (13-15). Although erlotinib as EGFR mutations targeted therapies has been considered for third-line therapy, the efficacy for the EGFR unselected or EGFR wild-type patients is unsatisfactory. Of the vascular endothelial growth factor (VEGF) family, VEGF and its receptors are considered to be the core signaling pathway in angiogenesis-related molecular mechanisms. Small molecule tyrosine kinase inhibitors (TKIs), especially the multi-target angiokinase inhibitors, have the capacity to potently and selectively inhibit the biochemical function of the downstream VEGFR-mediated signaling tyrosine kinases $(16,17)$. These multi-targeted oral TKIs that target angiogenesis pathways, including VEGFR, PDGFR, FGFR, c-KIT, FLT-3, etc., are consider to have a broad spectrum of inhibitory property on tumor angiogenesis and growth (17-20). At present, various anti-angiogenic drugs, such as apatinib, anlotinib, fruquintinib, lenvatinib have been applied and evaluated in many clinical studies for NSCLC. Thus, we perform this network analysis of randomized controlled trials reporting survival and safety data of those patients who have failed at least two lines of systemic therapy to determine the role of oral multi-target TKIs as third-line treatment in patient with advanced NSCLC.

\section{Methods}

\section{Study eligibility and identification}

We searched PubMed, Embase and Cochrane Central Register of Controlled Trials of the Cochrane Library respectively with a combination of the terms "non-small cell lung cancer (NSCLC)", "pretreated", "third-line therapy", "apatinib", "anlotinib", "fruquintinib", "lenvatinib" within the restriction limit of "randomized controlled trial" (by the cutoff of December 2018) to find out relevant studies, detailed search strategy was listed in Figure S1. We also reviewed abstract books and conducted a manual search of conference proceedings to make sure that the newest oncological progress enrolled. Besides, we also carefully checked and extracted all of the supplemental materials data from each trial to ensure no significant information was missing (Appendix 1). Moreover, we also re-examine the reference lists of related reviews as additional confirmation. The literature retrieval was conducted by two reviewers independently (F Lu and Z Zhang). Studies were included if they met our following inclusion criteria: (I) prospective phase II or III trials involving NSCLC patients; (II) patients received second or later-line therapy; (III) at least one available survival data regarding third-line treatment in advanced NSCLC patients. Studies that fail to meet the above criteria would be excluded from the network metaanalysis.

Outcomes measures, data extraction and quality assessment Therapeutic clinical outcomes including objective response 
rate (ORR), disease control rate (DCR), progression free survival (PFS), overall survival (OS) and toxicity outcomes including treatment related adverse event (TRAE), treatment related adverse event grade 3-5 (TRAE3-5), while specific concerned TRAE including hypertension, proteinuria, hand-foot skin reaction (HFSR), elevated ALT/ AST, nausea and vomiting, diarrhea were synthetically extracted. Study author, publication year, patient category, patients' ECOG, pathology, therapeutic regimens, the proportion of driver cancer-driver mutations, prior therapies, sample size and clinical outcomes mentioned above were reviewed and summed up by two investigators independently (F Lu and F Luo). The Cochrane Collaboration for Systematic Reviews guidelines were used to evaluate the quality of each study by two reviewers. The following seven items were assessed: random sequence generation, allocation sequence concealment, blinding of participants and personnel, blinding of the outcome assessment, incomplete outcome data, selective reporting, and other biases. The overall methodologic quality of each included study was assessed as "low risk of bias", "high risk of bias", or "unclear risk of bias" (21). Any dispute was resolved by discussion. All eligible studies were of high quality after the assessment.

\section{Statistical analyses}

We took the hazard ratio for survival outcomes (OS and PFS), and the odds ratio (OR) for binary outcomes (ORR, DCR, TRAE and TRAE 3-5 and specific concerned TRAE) as well as their $95 \%$ confidence intervals (CIs) to measure the efficacy and safety of various third-line regimens. For a specific comparison, an agent with $\mathrm{HR}$ for $\mathrm{OS}$ or PFS $<1$, or OR for ORR $>1$ was deemed preferable to its contrast in efficacy, while an agent with OR for TRAE or TRAE 3-5>1 was considered to have higher toxicity in the comparison. The network geometry was generated with plots depicting using StataMPversion 13.0 (StataCorp).

Firstly, the Bayesian network meta-analysis was performed with R v3.5.1 (gemtc package) (22) using a random-effects hierarchical model by assuming that different comparisons for each survival outcome (PFS, OS) shared a common heterogeneity parameter $(23,24) .95 \%$ CIs of the pooled HR excluding 1 or a two-sided $\mathrm{P}$ value less than 0.05 was considered statistically significant. Secondly, a random-effects network within a Bayesian framework was established using Markov chain Monte Carlo methods in ADDIS 1.15 (Drugis.org) (25). Thirdly, the binary clinical outcomes (ORR, DCR, TRAE, TRAEs 3-5 and specific concerned TRAE) network within studies was constructed and the relations among the odds ratios across studies were specified to make comparisons of different treatments in immunotherapy regimens. Fourthly, for each outcome, the probability of every agent being at each possible rank was estimated and presented. To be more intuitive, the surface under the cumulative ranking (SUCRA) curve was calculated based on the rank probability of each agent with corresponding outcomes to rank the efficacy and safety profile of various regimens (26) and displayed using the rank-heat plot (27) to provide a simple numerical summary for the relative ranking of the regimens. SUCRA value would be 1 if the agent is certain to be the best and 0 if it's certain to be the worst. For presentation of the intervention hierarchy, the SUCRA curve was used to rank the efficacy and safety outcomes (28). A rank-heat plot was used to depict the SUCRA values for all outcomes (27). Agents with colored green value in the rank-heat plot represented greater probabilities for better efficacy for efficacy outcomes; for toxicity outcomes, agents with colored red value in the rank-heat plot represented lower probabilities for incidence of specific adverse event.

The inconsistency within the multiple treatment comparison (MTC) was evaluated using a variance calculation such as Inconsistency Standard Deviation (ISD) and Random Effects Standard Deviation (RESD). The inclusive "1" in the $95 \%$ CI of ISD would indicate a low risk of inconsistency. The homogeneous results of RESD between consistency and inconsistency model would indicate a low risk of heterogeneity (28). The key parameters for evaluating inconsistency including direct, indirect and overall effects as well as ISD are listed in the Table S1. The node-splitting analysis would evaluate the consistency among different regimes if loop MTCs exist (29). The inconsistency within the network was evaluated by the variance calculation and node-splitting analysis using $\mathrm{R}$ v3.5.1, RStudio (gemtc package) and ADDIS 1.15. A P value $<0.05$ indicated significant inconsistency.

\section{Results}

\section{Characteristics of the selected studies}

One thousand and six hundred seventy-four original articles were identified through database searches and additional sources on 15 December 2018. After removing the duplicates and screening, 103 studies were considered 


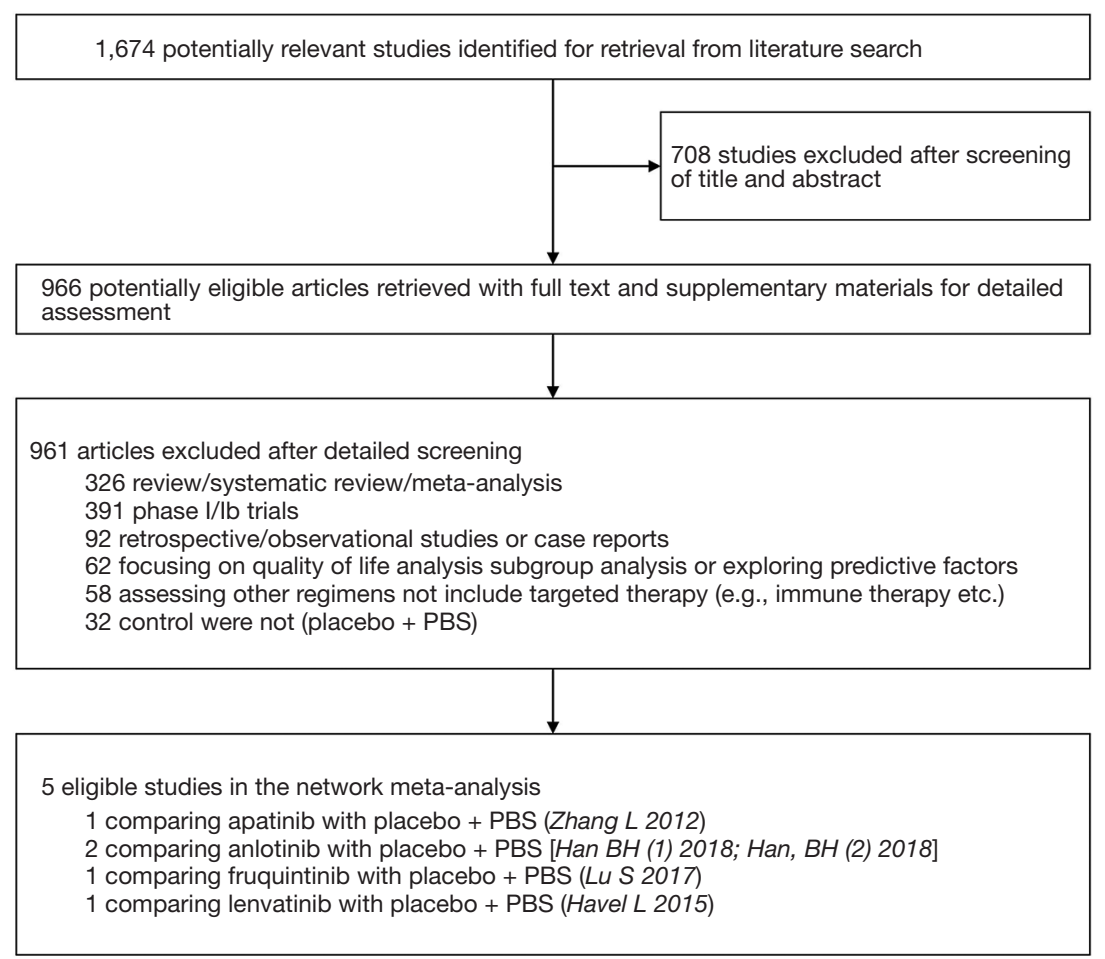

Figure 1 Flowchart of study selection and design.

potentially eligible for full-text assessment. Finally, five eligible studies were included in the network metaanalyses. Of the 98 excluded studies, 52 studies contained inappropriate patient/control groups, 31 lacked relevant interventions, 15 were non-RCTs. A flowchart following Preferred Reporting Items for Systematic Reviews and Meta-Analyses (PRISMA) is shown in Figure 1 and Appendix 2. Among these five phase II/III randomized control trials (30-34), Zhang [2012] compared apatinib with placebo as third-line treatment in NSCLC. Han [2017] and Han [2018] study investigated anlotinib and placebo in NSCLC. Lu [2017] performed trials comparing treatment with fruquintinib and placebo. And Havel [2015] compared lenvatinib with placebo in NSCLC third-line treatment. Detailed information about the characteristics of the studies included in this meta-analysis shown in Tables 1,S2.

\section{Risk of bias in included studies}

The judgments of the risk of bias in the five enrolled trials were perform by two investigators ( $\mathrm{Y}$ Zhang and $\mathrm{T}$ Zhou). Random sequence generation and allocation concealment were adequately performed in five studies. All studies required the blinding of participants and personnel, and were considered as having a low risk of attrition or reporting bias. Two trials blinded the process of outcome assessment while two trials had incomplete outcomes data. Detailed information can be found in Figure S2.

\section{Network geometry for MTCs}

Network diagrams was established for MTCs based on the included five RCTs (Figure 2). Solid lines between drugs represented the existence of direct comparisons. Outcome data for ORR, DCR, OS, PFS, TRAE, TRAE3-5, and specific concerned TRAE (hypertension, proteinuria, HFSR, elevated ALT/AST, nausea/vomiting, diarrhea) were synthetically extracted and analyzed. The five recommended treatments (apatinib, anlotinib, fruquintinib, lenvatinib and placebo plus PBS) were directed and indirect compared 


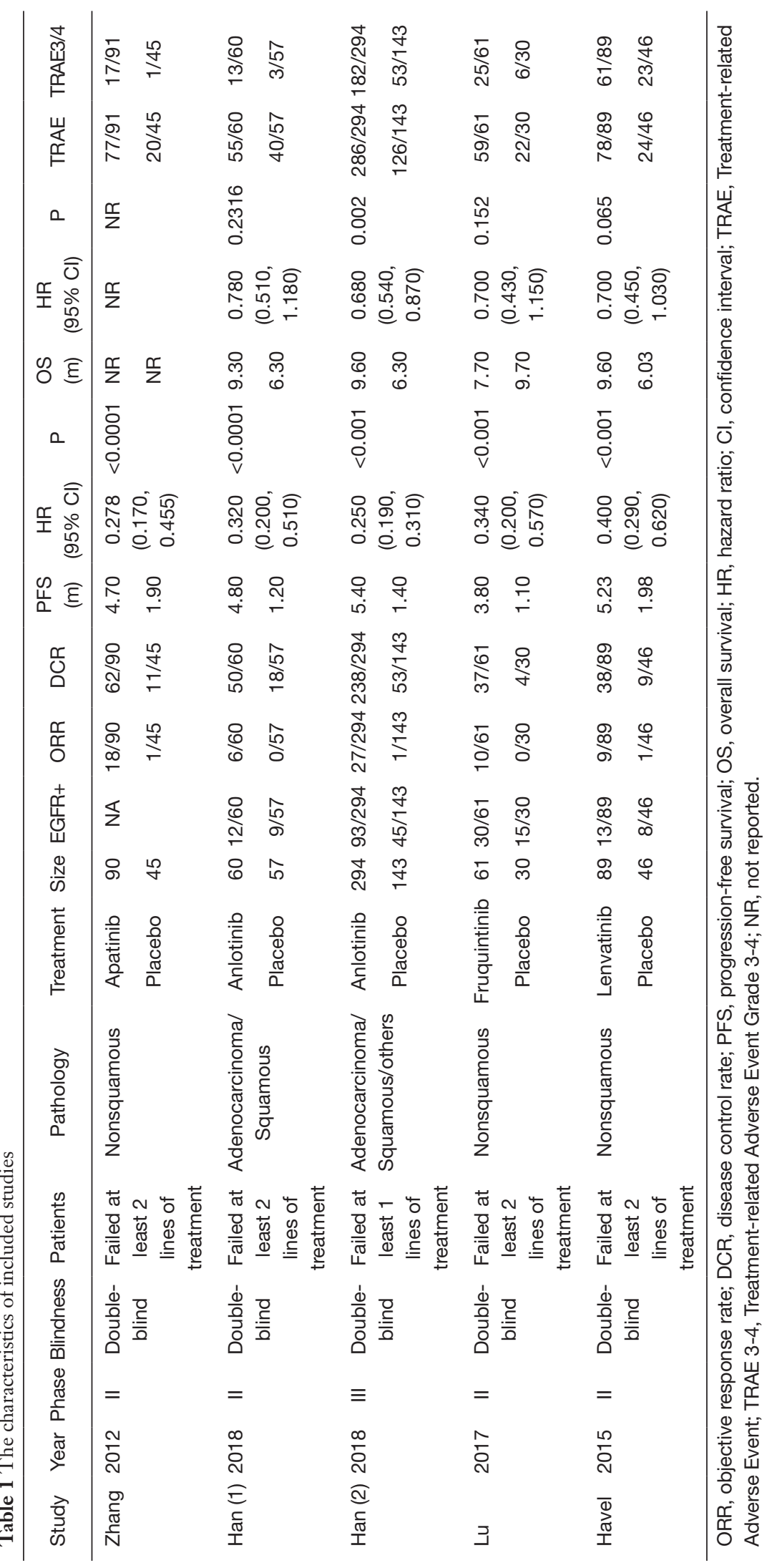




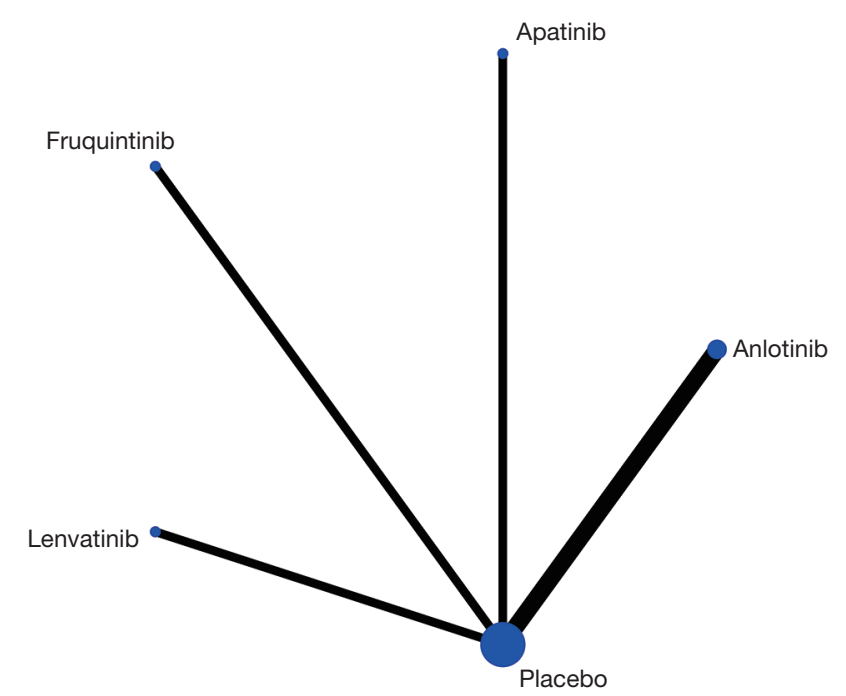

Figure 2 Network plot of multiple-target TKIs in third-line treatment of NSCLC. The size of each dot represents the number of patients receiving the corresponding intervention. The width of each line represents the number of studies of corresponding comparison. TKIs, tyrosine kinase inhibitors; NSCLC, non-small cell lung cancer.

against each other.

\section{Network meta-analyses for efficacy profile}

For ORR, DCR outcomes, the anlotinib and fruquintinib showed a significant improvement in ORR compared to placebo plus BSC $(\mathrm{OR}=39.26$; 95\% CI: 2.36-2,748.06; OR $=8.31 \times 10^{4} ; 95 \%$ CI: $\left.4.35-3.35 \times 10^{15}\right)$. The SUCRA rankings suggested fruquintinib (0.94) as the best intervention in ORR followed by anlotinib (0.63), apatinib (0.50), lenvatinib $(0.38)$ and placebo+BSC (0.05) which ranked last. For DCR, the result showed anlotinib has a significant improvement than placebo plus BSC (OR =8.69; 95\% CI: 1.70-50.18). The SUCRA rankings suggested fruquintinib (0.77) as the best intervention in DCR followed by anlotinib (0.71), apatinib (0.61), lenvatinib (0.37) and placebo+BSC (0.05) which ranked last.

For PFS, OS outcomes, according to the MTCs results, only anlotinib showed a significant improvement in PFS outcome when compared to placebo plus BSC (HR $=0.27 ; 95 \%$ CI, 0.10-0.78). The SUCRA rankings of PFS indicated that anlotinib (0.73) as the best intervention followed by apatinib (0.70), fruquintinib (0.57), lenvatinib
(0.46) and placebo+BSC (0.04). For OS outcomes, no statistically significant differences were observed in all these four interventions in this study, of which the HR of OS and 95\% CI existed marginal significant differences between anlotinib and placebo+BSC (HR $=0.71 ; 95 \%$ CI: 0.50-1.04). Taken OS rankings probability into account, the anlotinib is associated with the relatively higher SUCRA value (0.65), suggesting that the anlotinib may be superior to other treatments in survival outcomes. Additional data can be found in Figure 3 and Figure S3.

\section{Network meta-analyses for safety profile}

For TRAE, TRAE3-5 outcomes, MTCs showed insignificantly but higher risk of treatment-related adverse events with apatinib, anlotinib, fruquintinib, and lenvatinib in comparison to placebo plus BSC. The SUCRA values for these four TKIs were higher (range: 0.51-0.72) than for placebo plus BSC (0.04). Of the three highest ranking drugs (apatinib, fruquintinib, lenvatinib), the safety profile comparisons of drugs were no significant difference. Four trials were included in TRAE3-5 outcome analysis, anlotinib had the highest SUCRA value (0.75), among which however there was no statistically significant difference in TRAE3-5 across three high-ranking drugs.

For specific concerned TRAE, the incidence of hypertension during the treatment of multiple-target TKIs were reported in five trials. As demonstrated in Figures 4,S3, anlotinib is associated with the highest SUCRA rank value, while placebo plus BSC remained the lowest probability, with significantly higher rate of hypertension of the anlotinib group than in the placebo plus BSC group $(\mathrm{OR}=13.83$; 95\% CI: 1.64-183.71). Based on the results shown in Figures 4,S3, SUCRA value indicated that lenvatinib ranked the highest incidence of HFSR (0.86), followed by fruquintinib (0.8575). According to the analysis, the incidence of HFSR was significantly higher in the lenvatinib $\left(\mathrm{OR}=1.91 \times 10^{13} ; 95 \% \mathrm{CI}: 16.58-5.6 \times 10^{23}\right)$ and fruquintinib $\left(\mathrm{OR}=3.79 \times 10^{9} ; 95 \%\right.$ CI: $\left.394.44-1.22 \times 10^{20}\right)$ than in the placebo plus BSC group. Patients who received apatinib $\left(\mathrm{OR}=5.79 \times 10^{-9} ; 95 \% \mathrm{CI}: 2.23 \times 10^{-19}-0.11\right)$ or anlotinib $\left(\mathrm{OR}=3.02 \times 10^{-9} ; 95 \%\right.$ CI: $\left.7.63 \times 10^{-20}-0.04\right)$ regimen were less likely to suffer HFSR when compared with those treated with fruquintinib regimen. MTCs of other adverse events (proteinuria, elevated ALT/AST, nausea and vomiting, diarrhea) were also performed. Detailed results were shown 


\begin{tabular}{|c|c|c|c|c|}
\hline \multicolumn{5}{|l|}{ OR with $95 \% \mathrm{Cl}$ for ORR } \\
\hline \multicolumn{5}{|l|}{ Anlotinib } \\
\hline $2.41(0.02,1001.53)$ & Apatinib & & & \\
\hline $4.79 \times 10^{-4}\left(5.34 \times 10^{-15}, 17.81\right)$ & $1.52 \times 10^{-4}\left(1.43 \times 10^{-14}, 11.50\right)$ & Fruquintinib & & \\
\hline $5.87(0.03,1,687.86)$ & $2.51(0.01,690.91)$ & $1.52 \times 10^{4}\left(0.23,3.63 \times 10^{14}\right)$ & Lenvatinib & \\
\hline$\underline{39.26(2.36,2.748 .06)}$ & $15.93(0.44,1,140.26)$ & $\underline{8.31 \times 10^{4}\left(4.35,3.35 \times 10^{15}\right)}$ & $6.32(0.16,616.00)$ & Placebo + BSC \\
\hline \multicolumn{5}{|l|}{ OR with $95 \% \mathrm{Cl}$ for DCR } \\
\hline \multicolumn{5}{|l|}{ Anlotinib } \\
\hline $1.29(0.07,25.59)$ & Apatinib & & & \\
\hline $0.83(0.04,19.33)$ & $0.62(0.02,23.68)$ & Fruquintinib & & \\
\hline $2.54(0.15,56.96)$ & $1.99(0.07,71.22)$ & $3.22(0.09,122.22)$ & Lenvatinib & \\
\hline$\underline{8.69(1.70,50.18)}$ & $6.88(0.61,84.96)$ & $10.53(0.83,142.20)$ & $3.45(0.30,35.99)$ & Placebo + BSC \\
\hline \multicolumn{5}{|l|}{ HR with $95 \% \mathrm{Cl}$ for PFS } \\
\hline \multicolumn{5}{|l|}{ Anlotinib } \\
\hline $0.99(0.17,6.03)$ & Apatinib & & & \\
\hline $0.81(0.14,4.83)$ & $0.82(0.10,6.26)$ & Fruquintinib & & \\
\hline $0.69(0.12,4.01)$ & $0.69(0.09,5.33)$ & $0.85(0.11,6.61)$ & Lenvatinib & \\
\hline$\underline{0.27(0.10,0.78)}$ & $0.28(0.07,1.18)$ & $0.34(0.08,1.46)$ & $0.40(0.10,1.70)$ & Placebo + BSC \\
\hline \multicolumn{5}{|l|}{ HR with $95 \% \mathrm{Cl}$ for OS } \\
\hline \multicolumn{5}{|l|}{ Anlotinib } \\
\hline $1.01(0.49,2.12)$ & Fruquintinib & & & \\
\hline $1.01(0.51,2.05)$ & $1.00(0.42,2.39)$ & Lenvatinib & & \\
\hline $0.71(0.50,1.04)$ & $0.70(0.37,1.31)$ & $0.70(0.39,1.27)$ & Placebo+BSC & \\
\hline
\end{tabular}

Figure 3 Multiple treatment comparison for efficacy based on network consistency model. (OR $>1$ means the treatment in top left is better while HR >1 means the treatment in top left is worse). OR, odds ratio; HR, hazard ratio; CI, confidence interval; ORR, objective response rate; DCR, disease control rate; PFS, progression-free survival; OS, overall survival; BSC, best support care.

in Figure 3 and the SUCRA values were listed in Figure S3.

\section{Ranking probability}

The "ranking probabilities" produced by the network consistency model tended to indicate which multipletarget TKIs had higher ranking probability in all outcomes measured, including ORR, DCR, PFS, OS and TRAE etc. In general, regimens with greater ranking probabilities are more likely to produce better outcomes. Therefore, we calculated the SUCRA curve value with the primary ranking probabilities results to better represent the possible ranking and established a ranking heat plot to combine efficacy and safety profile based on the SUCRA. The SUCRA value of each regimen with corresponding outcomes are displayed in Figure $S 3$ and the synthesized ranking heat map plot was exhibited in Figure 4. Based on SUCRA value, the rank probabilities with cumulative value of being the most efficacious treatments were (ORR, DCR, PFS, OS): anlotinib $(0.50,0.61,0.73,0.65)$, apatinib $(0.63,0.71$, $0.70, \mathrm{NA})$, fruquintinib $(0.94,0.77,0.57,0.63)$, lenvatinib $(0.38,0.37,0.46,0.63)$ and placebo plus BSC $(0.05,0.05$, $0.04,0.09)$. According to SUCRA value of treatmentrelated adverse event, the results were (TRAE, TRAE35, hypertension, HFSR): anlotinib (0.61, 0.75, 0.57, 0.42), apatinib $(0.51, \mathrm{NA}, 0.69,0.32)$, fruquintinib $(0.72,0.65$, 


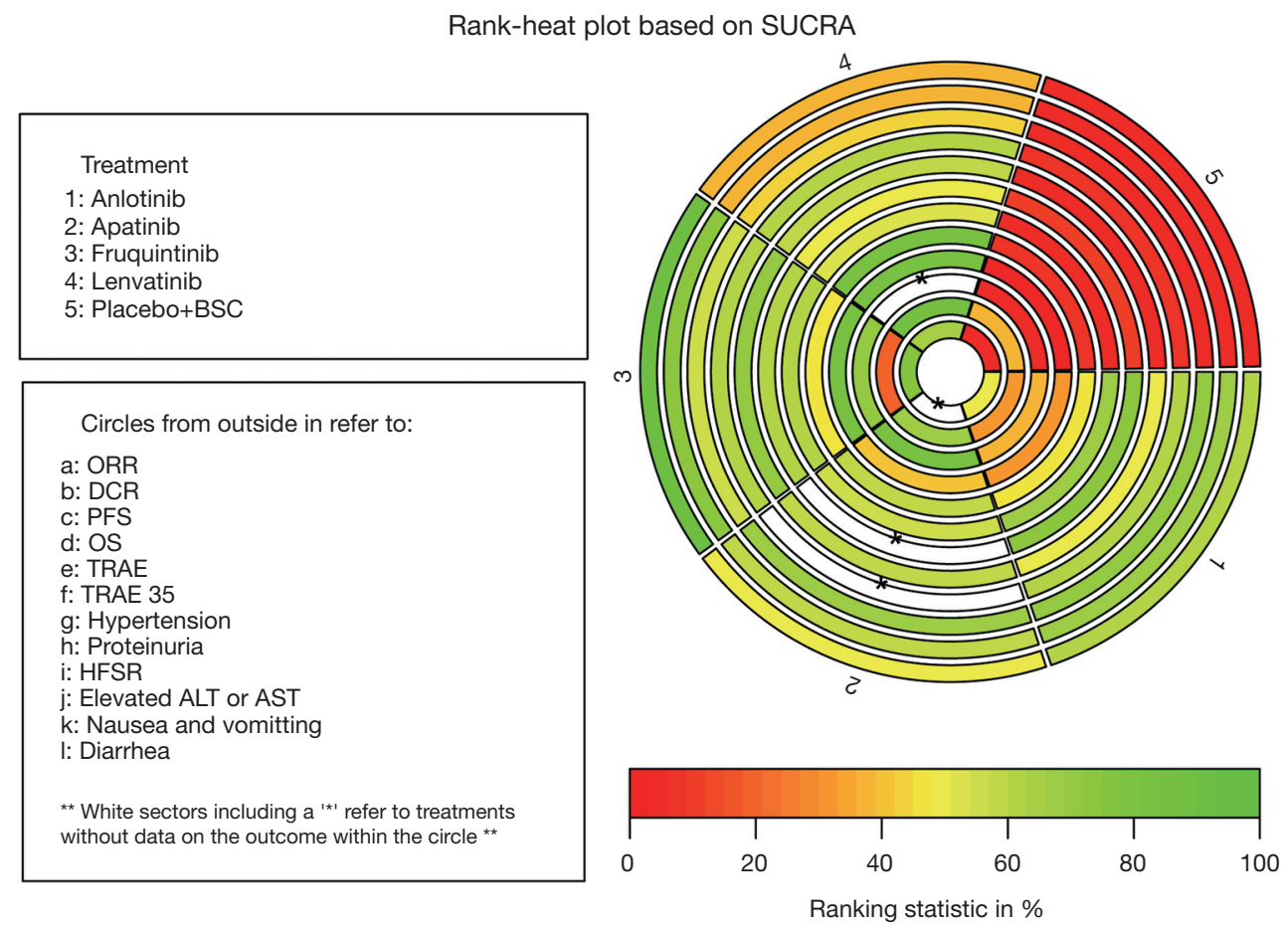

Figure 4 Rank-heat plot of the third-line regimens for NSCLC patients with efficacy and toxicity profile. Each sector is colored according to the surface under the cumulative ranking (SUCRA) value of the corresponding treatment and outcome. The scale consists of the transformation of three colors: red (0\%), yellow (50\%), and green (100\%), and each color is associated with a different pattern. Uncolored sectors show that the underlying treatment was not included in the network meta-analyses for the particular outcome. NSCLC, non-small cell lung cancer.

$0.64,0.86)$, lenvatinib $(0.61,0.51,0.56,0.86)$ and placebo plus BSC $(0.04,0.09,0.05,0.03)$ (more detail shown in Figure S3). As visualized in the ranking heat map plot in Figure 4, we could see that in general four multiple-target TKIs colored green in efficacy outcomes (ORR, DCR, PFS, and OS) and colored red in toxicity outcomes (e.g., TRAE, TRAE3-5, Hypertension, HFSR).

\section{Discussion}

Although a significant improvement in diagnostics and therapy for advanced NSCLC during the past decades, Availability of effective drugs for first- and secondline as well as maintenance therapy in NSCLC has led to increasing number of patients requiring third-line treatment. Clinicians inevitably encounter difficulty in treating patients with advanced NSCLC who experience a progression or relapse following first- and/or secondline treatment (35). For patients with advanced NSCLC, the paucity of approved agents for third-line therapy or beyond is becoming an important unmet medical need to be addressed. Considering the characteristics of patients failed at least 2 lines of systemic anticancer therapy, there is a great need for more effective therapies that improve clinical benefit with minimal toxicity (36). Theoretically, multitargeted-TKIs targeting various specific anti-cancer pathways are intended to be more effective with less toxicity, which provides a novel approach for cancer therapy. To our knowledge, this is the first network meta-analysis study to compare the efficacy and toxicity profile among different small molecule multi-targeted TKIs in third-line NSCLC treatment.

We conducted this comprehensive network meta-analysis with 5 RCTs included 915 advanced NSCLC patients to compare therapeutic efficacy and toxicity of four TKIs (apatinib, anlotinib, fruquintinib and lenvatinib) as thirdline treatment for advanced NSCLC. Overall, the results in this study demonstrated that anlotinib were superior in improving PFS compared to placebo plus BSC as third-line treatment in patients with advanced NSCLC after failure to 
prior therapy. As for OS, we found that three different TKIs (anlotinib, fruquintinib and lenvatinib) shared equivalent therapeutic effect without statistic differences. And notably anlotinib (as ranking the highest in SUCRA 0.65) seemed to have a marginal significant difference in contrast to placebo+BSC. Rank probabilities indicated that anlotinib potentially have a better efficacy compared with apatinib, fruquintinib and lenvatinib as third-line treatment for advanced NSCLC. Besides, the outcome of ORR, DCR and treatment-related adverse event were also analyzed. When compared with other agents, fruquintinib showed a better rank probability in SUCRA of ORR (0.94) and DCR (0.77), while potentially drug toxicity (TRAE, TRAE3-5) were observed in fruquintinib and anlotinib. For common TRAE, hypertension, proteinuria, HFSR, etc. were observed in all these TKIs. And fruquintinib and lenvatinib may relate to high rate of HFSR while anlotinib may relate to high risk of hypertension (detailed data can be found in Figure 5). All of these evidences indicated that these multi-targeted TKIs were potentially better in efficacy with tolerable toxicity in contrast to placebo as third-line treatment for advanced NSCLC. However, as in third-line therapy setting, OS may be considered an important outcome, we also provide some information on the potential of survival benefit that anlotinib may convey though the HR between anlotinib and placebo didn't reach statistic difference. More prospective randomized controlled trials or even real-world study specially comparing various novel agents as third-line therapy for advanced NSCLC are needed to validate the result.

Novel multi-target TKIs showed antitumor activity against tumor cells carrying mutations in VEGFR, PDGFR- $\alpha$, c-Kit, c-Met, Aurora-B, c-FMS, and discoidin domain receptor 1 (DDR1), which was a group of newly identified kinase targets involving the tumor progression (19,37-42). Inhibition of these targeted tyrosine kinases can prevent phosphorylation of the receptors and downstream signaling, which ultimately results in apoptosis of cancer cells (43). The potential to target multiple kinases may be an advantage over compound that have a single target axis, as the use of single-target TKIs (EGFR-TKI, ALK-TKI, etc.) commonly leads to tumor resistance and selected on the basis of the presence of driver mutations. Further, targeting the VEGF receptor alone may result in compensatory upregulation of other signal pathways, and leading to a resultant tumor progression. By inhibiting multi-arms of the signal pathway axis, multitargeted TKIs prevents more potential resistance to compounds that inhibit either target independently and may provide greater antitumor activity than targeting each pathway individually. In addition, multitargeted agents are directed at molecules associated with tumorigenesis, may provide greater efficacy and cause less overall toxicity than traditional cytotoxic drugs $(44,45)$. Further study is needed to explore the underlying potential mechanism and function of these agents.

There are several limitations of this study. First, although high quality of trials was included, only 915 patients were finally enrolled in this network meta-analysis, the sample size of this study was relatively small. We have taken this limitation into consideration before we interpreted these results with caution. Second, subgroup analysis was limited with lack of the individual patient data (IPD). We could not perform subgroup analysis stratified by subtypes of EGFR mutation status, race information and ECOG scores, which didn't reach to precise medical level. Third, here are not enough data for an in-depth analysis of OS (apatinib) and some other specific TRAE outcomes, more clinical data are warranted to support the survival benefit of multi-targeted oral TKIs. Since it's impossible to conduct further headto-head trials comparing efficacy and safety profile among these TKIs as third-line therapy. Our indirect comparative study helps clinicians get a general impression on the efficacy and safety of multi-targeted TKI as third-line treatment or beyond.

Recently, more and more novel compounds or their combination therapy such as proteasome inhibitor, histone deacetylase inhibitors and ICIs combined with antiangiogenic therapy are coming into sight and being evaluated in the process of clinical validation (46-48), bringing new hope for advanced NSCLC patients. Regardless of above limitations, this network meta-analysis preliminarily demonstrated the role of multi-targeted oral TKI as third-line therapy for NSCLC patients. The results led to important hints. Orally convenient TKIs, to some extent, could serve as a salvage therapy for those patients progress after front-line treatment in the hope of other upcoming novel therapy.

\section{Conclusions}

Our study indicated that multitarget TKIs (anlotinib, apatinib, fruquintinib and lenvatinib) have a promising therapeutic efficacy and acceptable toxicity profile and may consider to be an option as third-line treatment in NSCLC 


\begin{tabular}{|c|c|c|c|c|}
\hline OR with $95 \% \mathrm{Cl}$ for TRAE & & & & \\
\hline \multicolumn{5}{|l|}{ Anlotinib } \\
\hline $0.74(0.03,15.07)$ & Apatinib & & & \\
\hline $0.73(0.03,16.97)$ & $1.02(0.03,27.79)$ & $1.61(0.02,60.52)$ & Lenvatinib & \\
\hline $5.25(0.92,38.93)$ & $7.31(0.67,99.74)$ & $11.20(0.62,220.36)$ & $7.17(0.67,99.37)$ & Placebo + PBS \\
\hline \multicolumn{5}{|l|}{ OR with $95 \% \mathrm{Cl}$ for TRAE 3-5 } \\
\hline $1.13(0.11,12.97)$ & Fruquintinib & & & \\
\hline $1.49(0.20,15.67)$ & $1.33(0.10,19.14)$ & Lenvatinib & & \\
\hline $3.26(0.99,13.94)$ & $2.93(0.42,22.53)$ & $2.16(0.35,13.75)$ & Placebo + PBS & \\
\hline \multicolumn{5}{|c|}{ OR with $95 \% \mathrm{Cl}$ for Hypertension } \\
\hline \multicolumn{5}{|l|}{ Anlotinib } \\
\hline $13.83(1.64,183.71)$ & $9.73(0.42,299.49)$ & $13.35(0.36,1,369.93)$ & $9.60(0.39,268.16)$ & Placebo + PBS \\
\hline \multicolumn{5}{|l|}{ OR with $95 \% \mathrm{Cl}$ for Proteinuria } \\
\hline \multicolumn{5}{|l|}{ Anlotinib } \\
\hline $0.70(0.04,14.11)$ & Apatinib & & & \\
\hline $0.99(0.06,17.83)$ & $1.38(0.05,36.30)$ & Fruquintinib & & \\
\hline $0.27(0.02,5.23)$ & $0.39(0.01,11.29)$ & $0.27(0.01,9.71)$ & Lenvatinib & \\
\hline $2.61(0.51,12.85)$ & $3.69(0.34,36.11)$ & $2.63(0.25,29.02)$ & $9.21(0.78,113.85)$ & Placebo + PBS \\
\hline \multicolumn{5}{|l|}{ OR with $95 \% \mathrm{Cl}$ for $\mathrm{HFSR}$} \\
\hline \multicolumn{5}{|l|}{ Anlotinib } \\
\hline $0.39\left(1.36 \times 10^{-3}, 123.15\right)$ & Apatinib & & & \\
\hline $0.28(0.01,5.54)$ & $2.03(0.03,177.11)$ & Fruquintinib & & \\
\hline $2.09(0.46,11.52)$ & $14.75(0.92,602.41)$ & $7.48(0.68,128.76)$ & Placebo + PBS & \\
\hline \multicolumn{5}{|c|}{ OR with $95 \% \mathrm{Cl}$ for Nausea and Vomiting } \\
\hline \multicolumn{5}{|l|}{ Anlotinib } \\
\hline $0.23(0.01,8.43)$ & Apatinib & & & \\
\hline $1.40(0.04,45.63)$ & $6.14(0.15,272.96)$ & Fruquintinib & & \\
\hline $0.10\left(2.78 \times 10^{-3}, 3.60\right)$ & $0.42(0.01,18.05)$ & $0.07\left(1.9 \times 10^{-3}, 2.48\right)$ & Lenvatinib & \\
\hline $0.77(0.06,9.18)$ & $3.38(0.24,52.42)$ & $0.55(0.04,7.24)$ & $7.86(0.57,105.50)$ & Placebo + PBS \\
\hline \multicolumn{5}{|l|}{ OR with $95 \% \mathrm{Cl}$ for Diarrhea } \\
\hline \multicolumn{5}{|l|}{ Anlotinib } \\
\hline $0.32(0.01,7.41)$ & Fruquintinib & & & \\
\hline $0.56(0.04,8.44)$ & $1.84(0.05,124.07)$ & Lenvatinib & & \\
\hline $3.84(0.98,18.28)$ & $12.53(0.85,491.69)$ & $6.78(0.81,81.28)$ & Placebo + PBS & \\
\hline
\end{tabular}

Figure 5 Multiple treatment comparison for tolerability based on network consistency model. (OR $>1$ means the treatment in top left is worse). OR, odds ratio; CI, confidence interval; TRAE, Treatment-related Adverse Event; TRAE 3-5, Treatment-related Adverse Event Grade 3-5; HFSR, hand-foot skin reaction; BSC, best support care. 
patients.

\section{Clinical practice points}

* Four multi-targeted TKIs, including anlotinib, apatinib, fruquintinib and lenvatinib, are currently evaluated in third-line treatment of advanced NSCLC patients. However, there was no network meta-analysis among all the TKIs;

* Orally convenient multi-targeted TKIs, to some extent, could serve as a salvage therapy for those patients progress after front-line treatment in the hope of other upcoming subsequent therapy;

* These multitarget TKIs (anlotinib, apatinib, fruquintinib and lenvatinib) have a promising therapeutic efficacy and acceptable toxicity profile and may consider to be an option as third-line treatment in NSCLC patients.

\section{Acknowledgments}

Funding: This work was supported by National Key R\&D Program of China (Grant number: 2016YFC0905500, 2016YFC0905503), Science and Technology Program of Guangzhou (201607020031), Natural Science Foundation of Guangdong Province of China (Grant number: 2018A0303130243) and the 5010 Clinical Research Foundation of Sun Yat-sen University (Grant number: 2016001).

\section{Footnote}

Conflicts of Interest: The authors have no conflicts of interest to declare.

Ethical Statement: The authors are accountable for all aspects of the work in ensuring that questions related to the accuracy or integrity of any part of the work are appropriately investigated and resolved.

\section{References}

1. Ferlay J, Soerjomataram I, Dikshit R, et al. Cancer incidence and mortality worldwide: sources, methods and major patterns in GLOBOCAN 2012. Int J Cancer 2015;136:E359-86.

2. Novello S, Barlesi F, Califano R, et al. Metastatic nonsmall-cell lung cancer: ESMO Clinical Practice Guidelines for diagnosis, treatment and follow-up. Ann Oncol 2016;27:v1-27.

3. Iacono D, Chiari R, Metro G, et al. Future options for ALK-positive non-small cell lung cancer. Lung Cancer 2015;87:211-9.

4. Jemal A, Siegel R, Xu J, et al. Cancer statistics, 2010. CA Cancer J Clin 2010;60:277-300.

5. Ruffini E, Filosso PL, Bruna MC, et al. Recommended changes for $\mathrm{T}$ and $\mathrm{N}$ descriptors proposed by the International Association for the Study of Lung Cancer - Lung Cancer Staging Project: a validation study from a single-centre experience. Eur J Cardiothorac Surg 2009;36:1037-44.

6. Barnfield PC, Ellis PM. Second-Line Treatment of NonSmall Cell Lung Cancer: New Developments for Tumours Not Harbouring Targetable Oncogenic Driver Mutations. Drugs 2016;76:1321-36.

7. Leighl NB. Treatment paradigms for patients with metastatic non-small-cell lung cancer: first-, second-, and third-line. Curr Oncol 2012;19:S52-8.

8. Weiss JM, Stinchcombe TE. Second-Line Therapy for Advanced NSCLC. Oncologist 2013;18:947-53.

9. Ettinger DS, Wood DE, Aisner DL, et al. Non-Small Cell Lung Cancer, Version 5.2017, NCCN Clinical Practice Guidelines in Oncology. J Natl Compr Canc Netw 2017;15:504-35.

10. Gettinger S, Horn L, Jackman D, et al. Five-Year FollowUp of Nivolumab in Previously Treated Advanced NonSmall-Cell Lung Cancer: Results From the CA209-003 Study. J Clin Oncol 2018;36:1675-84.

11. Hensing TA, Schell MJ, Lee JH, et al. Factors associated with the likelihood of receiving second line therapy for advanced non-small cell lung cancer. Lung Cancer 2005;47:253-9.

12. Schiller JH, Harrington D, Belani CP, et al. Comparison of four chemotherapy regimens for advanced non-smallcell lung cancer. N Engl J Med 2002;346:92-8.

13. Hanahan D, Weinberg RA. The hallmarks of cancer. Cell 2000;100:57-70.

14. Hanahan D, Weinberg RA. Hallmarks of cancer: the next generation. Cell 2011;144:646-74.

15. Kerbel RS. Antiangiogenic therapy: a universal chemosensitization strategy for cancer? Science 2006;312:1171-5.

16. Pietras K, Hanahan D. A multitargeted, metronomic, and maximum-tolerated dose "chemo-switch" regimen is antiangiogenic, producing objective responses and survival benefit in a mouse model of cancer. J Clin Oncol 2005;23:939-52. 
17. Huang L, Huang Z, Bai Z, et al. Development and strategies of VEGFR-2/KDR inhibitors. Future Med Chem 2012;4:1839-52.

18. Knights V, Cook SJ. De-regulated FGF receptors as therapeutic targets in cancer. Pharmacol Ther 2010;125:105-17.

19. Turner N, Grose R. Fibroblast growth factor signalling: from development to cancer. Nat Rev Cancer 2010;10:116-29.

20. Wesche J, Haglund K, Haugsten EM. Fibroblast growth factors and their receptors in cancer. Biochem J 2011;437:199-213.

21. Higgins JP, Altman DG, Gotzsche PC, et al. The Cochrane Collaboration's tool for assessing risk of bias in randomised trials. Bmj 2011;343:d5928.

22. Chaimani A, Salanti G. Using network meta-analysis to evaluate the existence of small-study effects in a network of interventions. Res Synth Methods 2012;3:161-76.

23. Salanti G. Indirect and mixed-treatment comparison, network, or multiple-treatments meta-analysis: many names, many benefits, many concerns for the next generation evidence synthesis tool. Res Synth Methods 2012;3:80-97.

24. Salanti G, Higgins JP, Ades AE, et al. Evaluation of networks of randomized trials. Stat Methods Med Res 2008;17:279-301.

25. van Valkenhoef G, Tervonen T, Zwinkels T, et al. ADDIS: A decision support system for evidence-based medicine. Decis Support Syst 2013;55:459-75.

26. Salanti G, Ades AE, Ioannidis JP. Graphical methods and numerical summaries for presenting results from multipletreatment meta-analysis: an overview and tutorial. J Clin Epidemiol 2011;64:163-71.

27. Veroniki AA, Straus SE, Fyraridis A, et al. The rankheat plot is a novel way to present the results from a network meta-analysis including multiple outcomes. J Clin Epidemiol 2016;76:193-9.

28. White IR, Barrett JK, Jackson D, et al. Consistency and inconsistency in network meta-analysis: model estimation using multivariate meta-regression. Res Synth Methods 2012;3:111-25.

29. Dias S, Welton NJ, Sutton AJ, et al. Evidence synthesis for decision making 4: inconsistency in networks of evidence based on randomized controlled trials. Med Decis Making 2013;33:641-56.

30. Han B, Li K, Wang Q, et al. Effect of Anlotinib as a
Third-Line or Further Treatment on Overall Survival of Patients With Advanced Non-Small Cell Lung Cancer: The ALTER 0303 Phase 3 Randomized Clinical Trial. JAMA Oncol 2018;4:1569-75.

31. Han B, Li K, Zhao Y, et al. Anlotinib as a third-line therapy in patients with refractory advanced non-smallcell lung cancer: a multicentre, randomised phase II trial (ALTER0302). Br J Cancer 2018;118:654-61.

32. Havel L, Lee JS, Lee KH, et al. E7080 (lenvatinib) in addition to best supportive care (BSC) versus BSC alone in third-line or greater nonsquamous, non-small cell lung cancer (NSCLC). J Clin Oncol 2014;32:8043.

33. Lu S, Chang J, Liu X, et al. Randomized, DoubleBlind, Placebo-Controlled, Multicenter Phase II Study of Fruquintinib After Two Prior Chemotherapy Regimens in Chinese Patients With Advanced Nonsquamous NonSmall-Cell Lung Cancer. J Clin Oncol 2018;36:1207-17.

34. Zhang L, Shi M, Huang C, et al. A phase II, multicenter, placebo-controlled trial of apatinib in patients with advanced nonsquamous non-small cell lung cancer (NSCLC) after two previous treatment regimens. J Clin Oncol 2012;30:7548.

35. Syrigos KN, Saif MW, Karapanagiotou EM, et al. The need for third-line treatment in non-small cell lung cancer: an overview of new options. Anticancer Res 2011;31:649-59.

36. Asahina H, Sekine I, Horinouchi H, et al. Retrospective analysis of third-line and fourth-line chemotherapy for advanced non-small-cell lung cancer. Clin Lung Cancer 2012;13:39-43.

37. Ambrogio C, Gomez-Lopez G, Falcone M, et al. Combined inhibition of DDR1 and Notch signaling is a therapeutic strategy for KRAS-driven lung adenocarcinoma. Nat Med 2016;22:270-7.

38. Ashton S, Song YH, Nolan J, et al. Aurora kinase inhibitor nanoparticles target tumors with favorable therapeutic index in vivo. Sci Transl Med 2016;8:325ra17.

39. Rosell R, Karachaliou N. Large-scale screening for somatic mutations in lung cancer. Lancet 2016;387:1354-6.

40. Strumberg D, Richly H, Hilger RA, et al. Phase I clinical and pharmacokinetic study of the Novel Raf kinase and vascular endothelial growth factor receptor inhibitor BAY 43-9006 in patients with advanced refractory solid tumors. J Clin Oncol 2005;23:965-72. 
41. Sun Y, Niu W, Du F, et al. Safety, pharmacokinetics, and antitumor properties of anlotinib, an oral multitarget tyrosine kinase inhibitor, in patients with advanced refractory solid tumors. J Hematol Oncol 2016;9:105.

42. Wang C, Chen J, Cao W, et al. Aurora-B and HDAC synergistically regulate survival and proliferation of lymphoma cell via AKT, mTOR and Notch pathways. Eur J Pharmacol 2016;779:1-7.

43. Hait WN, Hambley TW. Targeted cancer therapeutics. Cancer Res 2009;69:1263-7; discussion 7.

44. Ballas MS, Chachoua A. Rationale for targeting VEGF, FGF, and PDGF for the treatment of NSCLC. Onco Targets Ther 2011;4:43-58.

45. Langer CJ, Mok T, Postmus PE. Targeted agents in the third-/fourth-line treatment of patients with advanced

Cite this article as: Zhang Z, Zhao Y, Lu F, Hou X, Ma Y, Luo F, Zeng K, Zhao S, Zhang Y, Zhou T, Yang Y, Fang W, Huang Y, Zhang L, Zhao H. Multi-targeted tyrosine kinase inhibitors as third-line regimen in advanced non-small cell lung cancer: a network meta-analysis. Ann Transl Med 2019;7(18):452. doi: 10.21037/atm.2019.08.51 (stage III/IV) non-small cell lung cancer (NSCLC).

Cancer Treat Rev 2013;39:252-60.

46. Garassino MC, Cho BC, Kim JH, et al. Durvalumab as third-line or later treatment for advanced non-small-cell lung cancer (ATLANTIC): an open-label, single-arm, phase 2 study. Lancet Oncol 2018;19:521-36.

47. Hoang T, Campbell TC, Zhang C, et al. Vorinostat and bortezomib as third-line therapy in patients with advanced non-small cell lung cancer: a Wisconsin Oncology Network Phase II study. Invest New Drugs 2014;32:195-9.

48. Xing P, Zhu Y, Shan L, et al. The role of weekly nanoparticle albumin bound paclitaxel monotherapy as second line or later treatment for advanced NSCLC in China. Oncotarget 2017;8:87442-54. 


\section{Appendix 1}

\begin{tabular}{|c|c|}
\hline Section/topic & \# Checklist item \\
\hline
\end{tabular}

\section{TITLE}

Title

ABSTRACT

Structured summary

INTRODUCTION

Rationale

Objectives

METHODS

Protocol and registration

Eligibility criteria

Information sources

Search

Study selection

Data collection process

Data items

Risk of bias in individual studies

Summary measures

Synthesis of results
Multi-targeted tyrosine kinase inhibitors as third-line regimen in advanced non-small cell lung cancer: a network meta-analysis

2 Background: Four multi-targeted tyrosine kinase inhibitors (TKIs) including apatinib, anlotinib, fruquintinib and lenvatinib are currently available as third-line regimen for advanced non-small cell lung cancer (NSCLC) patients who failed at least two lines of systemic therapy. Limited evidence was provided to demonstrate the general efficacy and safety profile of these drugs as third-line treatment approach for NSCLC.

Methods: Eligible literature was searched from electronic database. Data of objective response rate (ORR), disease control rate (DCR), progression-free survival (PFS), overall survival (OS), treatment related adverse event (TRAE), treatment related adverse event grade 3-5 (TRAE3-5), hypertension, proteinuria, hand-foot skin reaction (HFSR), elevated ALT/AST, nausea and vomiting, diarrhea were synthetically extracted. Multiple-treatments comparisons (MTCs) based on a Bayesian consistency model integrated the efficacy and toxicity outcomes. Rank probabilities of each regimen were assessed and clustered by the surface under the cumulative ranking curve.

Results: Five phase II/III randomized trials involving 915 advanced NSCLC patients were enrolled. MTCs showed that four multi-targeted TKIs shared equivalent efficacy in terms of outcome measures, of which anlotinib stood out in ORR (OR =39.26; 95\% Cl: 2.36-2,748.06), DCR (OR =8.69; 95\% Cl: 1.70-50.18) and PFS (HR $=0.27 ; 95 \% \mathrm{Cl}: 0.10-0.78$ ) when compared with placebo plus BSC. No significantly differences were observed among these TKIs and placebo with respect to OS, TRAE and TRAE 3-5. Fruquintinib and lenvatinib may relate to high rate of HFSR while anlotinib may relate to hypertension.

Conclusions: Multi-targeted TKIs (apatinib, anlotinib, fruquintinib and lenvatinib) with acceptable efficacy and safety profile were options for advanced NSCLC in thirdline setting.

3 The emergence of ICls for the treatment of malignancy has significantly changed the therapeutic landscape of lung cancer, especially NSCLC. These encouraging therapies have improved survival and the quality of life for NSCLC patients, however, most of the patients experience disease progression after receiving a certain period of time with first- or second-line therapy and there is currently no standard therapeutic options beyond second-line, placing the subsequent salvage therapy in a difficult situation.

4 At present, various anti-angiogenic drugs, such as apatinib, anlotinib, fruquintinib, lenvatinib have been applied and evaluated in many clinical studies for NSCLC. Thus we perform this network analysis of randomized controlled trials reporting survival and safety data of those patients who have failed at least two lines of systemic therapy to determine the role of oral multi-target TKIs as third-line treatment in patient with advanced NSCLC.

5 Not needed

6 (I) prospective phase II or III trials involving NSCLC patients; (II) patients received second or later-line therapy; (III) at least one available survival data regarding third-line treatment in advanced NSCLC patients.

7 Random control trials (RCTs) by the cutoff of December 2018 in the PubMed, Embase, Cochrane Central Register of Controlled Trials databases were searched. An additional search through conference abstracts, reference lists of selected trials, relevant previous systematic reviews and meta-analysis were also screened.

8 A combination of the terms a combination of the terms "non-small cell lung cancer (NSCLC)", "pretreated", "third-line therapy", "apatinib", "anlotinib", "fruquintinib", "lenvatinib" within the restriction limit of "randomized controlled trial" were used to find relevant articles and ensure the latest research progress enrolled. Detailed search strategy was listed in Figure S1. We also reviewed abstract books and conducted a manual search of conference proceedings to make sure that the newest oncological progress enrolled. Besides, we also carefully checked and extracted all of the supplemental materials data from each trial to ensure no significant information was missing. Moreover, we also re-examine the reference lists of related reviews as additional confirmation. The literature retrieval was conducted by two reviewers independently (F Lu and Z Zhang).

9 Eligible studies should meet the following criteria: (I) prospective phase II or III trials involving NSCLC patients; (II) patients received second or later-line therapy; (III) at least one available survival data regarding third-line treatment in advanced NSCLC patients.

10 Study author, publication year, patient category, pathology, therapeutic regimens, sample size and clinical outcomes mentioned above were reviewed and summed up by two investigators independently. ( F Lu and F Luo) to assess the eligibility. Disagreements were discussed with a third author ( $\mathrm{Z}$ Zhang) to reach a consensus. The same authors used a standardized form to extract data independently.

11 Therapeutic clinical outcomes including objective response rate (ORR), disease control rate (DCR), progression free survival (PFS), overall survival (OS) and toxicity outcomes including treatment related adverse event (TRAE), treatment related adverse event grade 3-5 (TRAE3-5), while specific concerned TRAE including hypertension, proteinuria, hand-foot skin reaction (HFSR), elevated ALT/AST, nausea and vomiting, diarrhea were synthetically extracted.

12 The Cochrane Collaboration for Systematic Reviews guidelines were used to evaluate the quality of each study by two reviewers. The following seven items were assessed random sequence generation, allocation sequence concealment, blinding of participants and personnel, blinding of the outcome assessment, incomplete outcome data, selective reporting, and other biases. The overall methodologic quality of each included study was assessed as "low risk of bias", "high risk of bias", or "unclear risk of bias".

13 Firstly, the Bayesian network meta-analysis was performed with R v3.5.1 (gemtc package) (22) using a random-effects hierarchical model by assuming that different comparisons for each survival outcome (PFS, OS) shared a common heterogeneity parameter (23,24). 95\% Cls of the pooled HR excluding 1 or a two-sided P value less than 0.05 was considered statistically significant. Secondly, a random-effects network within a Bayesian framework was established using Markov chain Monte Carlo methods in ADDIS 1.15 (Drugis.org) (25). Thirdly, the binary clinical outcomes (ORR, DCR, TRAE, TRAEs 3-5 and specific concerned TRAE) network within studies was constructed and the relations among the odds ratios across studies were specified to make comparisons of different treatments in immunotherapy regimens. Fourthly, for each outcome, the probability of every agent being at each possible rank was estimated and presented. To be more intuitive, the surface under the cumulative ranking (SUCRA) curve was calculated based on the rank probability of each agent with corresponding outcomes to rank the efficacy and safety profile of various regimens (26) and displayed using the rank-heat plot (27) to provide a simple numerical summary for the relative ranking of the regimens. SUCRA value would be 1 if the agent is certain to be the best and 0 if it's certain to be the worst.

14 We took the hazard ratio for survival outcomes (OS and PFS), and the odds ratio (OR) for binary outcomes (ORR, DCR, TRAE and TRAE 3-5 and specific concerned TRAE) as well as their $95 \%$ confidence intervals (Cls) to measure the efficacy and safety of various third-line regimens. For a specific comparison, an agent with $\mathrm{HR}$ for OS or PFS $<1$, or OR for ORR $>1$ was deemed preferable to its contrast in efficacy, while an agent with OR for TRAE or TRAE 3-5 $>1$ was considered to have higher toxicity in the comparison. The network geometry was generated with plots depicting using StataMPversion 13.0 (StataCorp). 


\section{Section/topic
Risk of bias across studies}

Additional analyses

RESULTS

Study selection

Study characteristics

Risk of bias within studies

Results of individual studies

Synthesis of results

Risk of bias across studies Additional analysis

DISCUSSION

Summary of evidence

Limitations

Conclusions

FUNDING

Funding
15 The details of risk of bias for included studies could be found in Figure S2.

16 The inconsistency within the multiple treatment comparison (MTC) was evaluated using a variance calculation such as Inconsistency Standard Deviation (ISD) and Random Effects Standard Deviation (RESD). The inclusive "1" in the 95\% Cl of ISD would indicate a low risk of inconsistency. The homogeneous results of RESD between consistency and inconsistency model would indicate a low risk of heterogeneity. The key parameters for evaluating inconsistency including direct, indirect and overall effects as well as ISD are listed in the Table S1. The node-splitting analysis would evaluate the consistency among different regimes if loop MTCs exist. The inconsistency within the network was evaluated by the variance calculation and node-splitting analysis using R v3.5.1, RStudio (gemtc package) and ADDIS 1.15. A P value $<0.05$ indicated significant inconsistency.

One thousand and six hundred seventy-four original articles were identified through database searches and additional sources on 15 December 2018 . After removing the duplicates and screening, 103 studies were considered potentially eligible for full-text assessment. Finally, five eligible studies were included in the network meta-analyses. Of the 98 excluded studies, 52 studies contained inappropriate patient/control groups, 31 lacked relevant interventions, 15 were non-RCTs. A flowchart following Preferred Reporting Items for Systematic Reviews and Meta-Analyses (PRISMA) is shown in Figure 1.

18 Among these five phase I//II randomized control trials (30-34), Zhang [2012] compared apatinib with placebo as third-line treatment in NSCLC. Han [2017] and Han [2018] study investigated anlotinib and placebo in NSCLC. Lu [2017] performed trials comparing treatment with fruquintinib and placebo. And Havel [2015] compared lenvatinib with placebo in NSCLC third-line treatment. Detailed information about the characteristics of the studies included in this meta-analysis shown in Table 1.

19 The details of risk of bias for included studies could be found in Figure S2.

20 Table 1 summarized the characteristics of all included studies, such as trial name, chemotherapy category, antiemetic regimens, the dose of olanzapine and clinical outcomes.

21 Network meta-analyses for efficacy profile

For ORR, DCR outcomes, the anlotinib and fruquintinib showed a significant improvement in ORR compared to placebo plus BSC (OR $=39.26$; $95 \%$ Cl: $2.36-2.75 \times 10^{3}$; $\left.\mathrm{OR}=8.31 \times 10^{4} ; 95 \% \mathrm{Cl}: 4.35-3.35 \times 10^{15}\right)$. The SUCRA rankings suggested fruquintinib $(0.94)$ as the best intervention in ORR followed by anlotinib $(0.63)$, apatinib $(0.50)$, lenvatinib (0.38) and placebo+BSC (0.05) which ranked last. For DCR, the result showed anlotinib has a significant improvement than placebo plus BSC (OR $=8.69$; $95 \% \mathrm{Cl}: 1.7-50.18)$. The SUCRA rankings suggested fruquintinib $(0.77)$ as the best intervention in DCR followed by anlotinib $(0.71)$, apatinib $(0.61)$, lenvatinib $(0.37)$ and placebo+BSC (0.05) which ranked last.

For PFS, OS outcomes, according to the MTCs results, only anlotinib showed a significant improvement in PFS outcome when compared to placebo plus $\mathrm{BSC}(\mathrm{HR}=0.27 ; 95 \% \mathrm{Cl}, 0.1-0.78)$. The SUCRA rankings of PFS indicated that anlotinib $(0.73)$ as the best intervention followed by apatinib $(0.70)$, fruquintinib $(0.57)$, lenvatinib (0.46) and placebo+BSC (0.04). For OS outcomes, no statistically significant differences were observed in all these four interventions in this study, of which the HR of OS and $95 \% \mathrm{Cl}$ existed marginal significant differences between anlotinib and placebo+BSC (HR =0.71; 95\% Cl: 0.50-1.04). Taken OS rankings probability into account, the anlotinib is associated with the relatively higher SUCRA value (0.65), suggesting that the anlotinib may be superior to other treatments in survival outcomes. Additional data can be found in Figure 3 and Figure S3.

\section{Network meta-analyses for safety profile}

For TRAE, TRAE3-5 outcomes, MTCs showed insignificantly but higher risk of treatment-related adverse events with apatinib, anlotinib, fruquintinib, and lenvatinib in comparison to placebo plus BSC. The SUCRA values for these four TKIs were higher (range: 0.51-0.72) than for placebo plus BSC (0.04). Of the three highest ranking drugs (apatinib, fruquintinib, lenvatinib), the safety profile comparisons of drugs were no significant difference. Four trials were included in TRAE3-5 outcome analysis, anlotinib had the highest SUCRA value (0.75), among which however there was no statistically significant difference in TRAE3-5 across three high-ranking drugs.

For specific concerned TRAE, the incidence of hypertension during the treatment of multiple-target TKIs were reported in five trials. As demonstrated in Figures 4,S3, anlotinib is associated with the highest SUCRA rank value, while placebo plus BSC remained the lowest probability, with significantly higher rate of hypertension of the anlotinib group than in the placebo plus BSC group (OR=13.83; 95\% Cl: 1.64-183.71). Based on the results shown in Figures 4,S3, SUCRA value indicated that lenvatinib ranked the highest incidence of HFSR $(0.86)$, followed by fruquintinib $(0.8575)$. According to the analysis, the incidence of HFSR was significantly higher in the lenvatinib $\left(\mathrm{OR}=1.91 \times 10^{13} ; 95 \% \mathrm{Cl}: 16.58-5.6 \times 10^{23}\right)$ and fruquintinib $\left(\mathrm{OR}=3.79 \times 10^{9} ; 95 \% \mathrm{Cl}: 394.44-1.22 \times 10^{20}\right)$ than in the placebo plus BSC group. Patients who received apatinib (OR $\left.=5.79 \times 10^{-9} ; 95 \% \mathrm{Cl}: 2.23 \times 10^{-19}-0.11\right)$ or anlotinib $\left(\mathrm{OR}=3.02 \times 10^{-9} ; 95 \% \mathrm{Cl}: 7.63 \times 10^{-20}-0.04\right)$ regimen were less likely to suffer $\mathrm{HFSR}$ when compared with those treated with fruquintinib regimen. MTCs of other adverse events (proteinuria, elevated ALT/AST, nausea and vomiting, diarrhea) were also performed. Detailed results were shown in Figure 3 and the SUCRA values were listed in Figure S3.

22 The details of risk of bias for included studies could be found in Figure $S 2$.

23 Ranking probability

The "ranking probabilities" produced by the network consistency model tended to indicate which multiple-target TKls had higher ranking probability in all outcomes measured, including ORR, DCR, PFS, OS and TRAE etc. In general, regimens with greater ranking probabilities are more likely to produce better outcomes. Therefore, we calculated the SUCRA curve value with the primary ranking probabilities results to better represent the possible ranking and established a ranking heat plot to combine efficacy and safety profile based on the SUCRA. The SUCRA value of each regimen with corresponding outcomes are displayed in Figure S3 and the synthesized ranking heat map plot was exhibited in Figure 4.

24 To our knowledge, this is the first network meta-analysis study to compare the efficacy and toxicity profile among different small molecule multi-targeted TKls in thirdline NSCLC treatment. We conducted this comprehensive network meta-analysis with 5 RCTs included 915 advanced NSCLC patients to compare therapeutic efficacy and toxicity of four TKIs (apatinib, anlotinib, fruquintinib and lenvatinib) as third-line treatment for advanced NSCLC. Overall, the results in this study demonstrated that anlotinib were superior in improving PFS compared to placebo plus BSC as third-line treatment in patients with advanced NSCLC after failure to prior therapy. As for OS, we found that three different TKIs (anlotinib, fruquintinib and lenvatinib) shared equivalent therapeutic effect without statistic differences. And notably anlotinib (as ranking the highest in SUCRA 0.65) seemed to have a marginal significant difference in contrast to placebo+BSC. Rank probabilities indicated that anlotinib potentially have a better efficacy compared with apatinib, fruquintinib and lenvatinib as third-line treatment for advanced NSCLC. Besides, the outcome of ORR, DCR and treatment-related adverse event were also analyzed. When compared with other agents, fruquintinib showed a better rank probability in SUCRA of ORR (0.94) and DCR (0.77), while potentially drug toxicity (TRAE, TRAE3-5) were observed in fruquintinib and anlotinib. For common TRAE, hypertension, proteinuria, HFSR, etc. were observed in all these TKIs. And fruquintinib and lenvatinib may relate to high rate of HFSR while anlotinib may relate to high risk of hypertension (detailed data can be found in Figure 5). All of these evidences indicated that these multi-targeted TKIs were potentially better in efficacy with tolerable toxicity in contrast to placebo as thirdline treatment for advanced NSCLC. However, as in third-line therapy setting, OS may be considered an important outcome, we also provide some information on the potential of survival benefit that anlotinib may convey though the HR between anlotinib and placebo didn't reach statistic difference. More prospective randomized controlled trials or even real-world study specially comparing various novel agents as third-line therapy for advanced NSCLC are needed to validate the result.

25 There are several limitations of this study. First, although high quality of trials was included, only 915 patients were finally enrolled in this network meta-analysis, the sample size of this study was relatively small. Second, subgroup analysis was limited with lack of the individual patient data (IPD). We could not perform subgroup analysis stratified by subtypes of EGFR mutation status, race information, which didn't reach to precise medical level. Third, here are not enough data for an in-depth analysis of OS (apatinib) and some other specific TRAE outcomes, more clinical data are warranted to support the survival benefit of multi-targeted oral tyrosine kinase inhibitors.

26 Regardless of above limitations, this network meta-analysis preliminarily demonstrated the role of multi-targeted oral TKI as third-line therapy for NSCLC patients. The results led to important hints. Orally convenient TKIs, to some extent, could serve as a salvage therapy for those patients progress after front-line treatment in the hope of other upcoming novel therapy. In conclusion, our study indicated that multitarget TKIs (anlotinib, apatinib, fruquintinib and lenvatinib) have a promising therapeutic efficacy and acceptable toxicity profile and may consider to be an option as third-line treatment in NSCLC patients.

27 This work was supported by National Key R\&D Program of China (Grant number: 2016YFC0905500, 2016YFC0905503), Science and Technology Program of Guangzhou (201607020031), Natural Science Foundation of Guangdong Province of China (Grant number: 2018A0303130243) and the 5010 Clinical Research Foundation of Sun Yat-sen University (Grant number: 2016001).

From: Moher D, Liberati A, Tetzlaff J, et al. Preferred Reporting Items for Systematic Reviews and Meta-Analyses: The PRISMA Statement. PLoS Med 2009;6:e1000097. doi:10.1371/journal.pmed1000097

For more information, visit: www.prisma-statement.org. 
Appendix 2

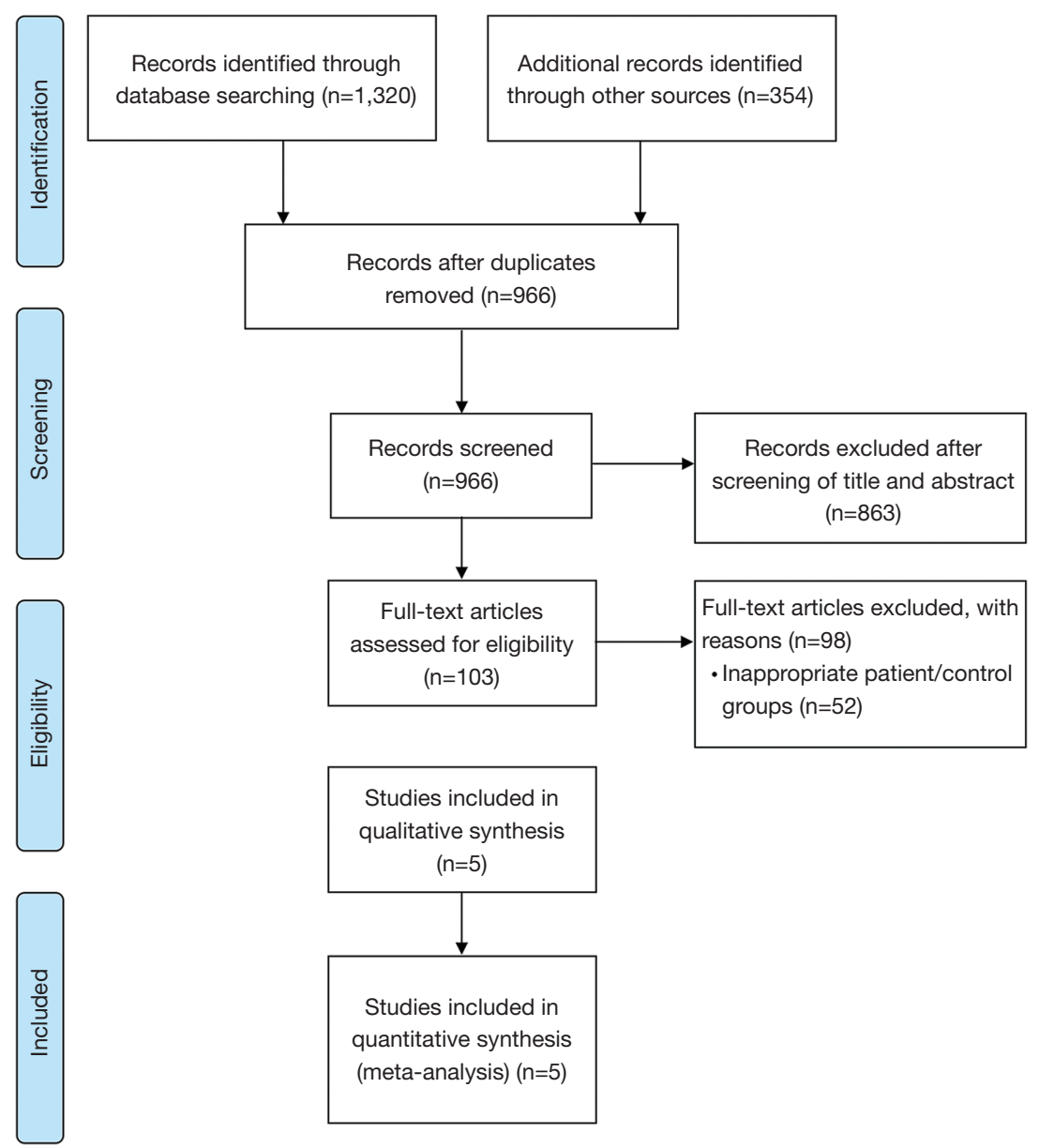




\begin{tabular}{|c|c|c|}
\hline Lung cancer & $\# 1$ & $\begin{array}{l}\text { (((((lung neoplasms[MeSH]) OR “Carcinoma, Non-Small-Cell Lung”[Mesh]) OR lung carcinom*[tiab]) OR lung } \\
\text { neoplasm*[tiab]) OR lung cancer[tiab]) OR NSCLC[tiab]) OR non small cell lung[tiab] }\end{array}$ \\
\hline Advanced & $\# 2$ & $\begin{array}{l}\text { ((((((“Neoplasm Metastasis”[MeSH]) OR advanced[tiab]) OR stage IV[tiab]) OR stage 4[tiab]) OR metastases[tiab]) OR } \\
\text { metastatic[tiab]) OR stage four[tiab] }\end{array}$ \\
\hline Intervention & $\# 3$ & $\begin{array}{l}\text { ((((((“"erlotinib”[Supplementary Concept]) OR tarceva[tiab]) OR erlotinib[tiab])) OR ((Apatinib[tiab]) } \\
\text { OR”Apatinib”[Supplementary Concept])) OR ((Lenvatinib[tiab] OR “Lenvatinib”[Supplementary Concept])) OR } \\
((\text { Fruquintinib[tiab]) OR “Fruquintinib”[Supplementary Concept])) OR ((“Anlotinib”[Supplementary Concept]) OR } \\
\text { Anlotinib[tiab]) }\end{array}$ \\
\hline Third line & $\# 4$ & $\begin{array}{l}(((((\text { third line[tiab]) OR phase } ॥[\text { tiab]) OR phase III[tiab]) OR pretreat*[tiab]) OR previously treated[tiab]) OR } \\
\text { refractory[tiab]) OR recurrent[tiab] }\end{array}$ \\
\hline RCT & $\# 5$ & $\begin{array}{l}(((((((\text { randomized controlled trial[Publication Type]) OR controlled clinical trial[Publication Type]) OR randomized[tiab]) } \\
\text { OR placebo[tiab]) OR drug therapy[sh]) OR randomly[tiab]) OR trial[tiab]) OR groups[tiab]) NOT ((animals[mh]) NOT } \\
\text { humans[mh]) }\end{array}$ \\
\hline
\end{tabular}

Figure S1 Search strategy.

Table S1 Evaluation of inconsistency (random effects standard deviation and inconsistency standard deviation)

\begin{tabular}{lccc}
\hline Outcomes & $\begin{array}{c}\text { Random effects standard deviation } \\
\text { (consistency model) }\end{array}$ & $\begin{array}{c}\text { Random effects standard deviation } \\
\text { (inconsistency model) }\end{array}$ & $\begin{array}{c}\text { Inconsistency standard deviation } \\
\text { (ISD) }\end{array}$ \\
\hline ORR & $1.27(0.08,2.55)$ & $1.25(0.08,2.54)$ & $1.29(0.06,2.56)$ \\
DCR & $0.67(0.01,2.20)$ & $0.68(0.04,2.19)$ & $1.14(0.06,2.27)$ \\
TRAE & $0.81(0.05,2.10)$ & $0.71(0.04,2.08)$ & $1.10(0.05,2.15)$ \\
TRAE3-5 & $0.60(0.05,1.43)$ & $0.59(0.03,1.42)$ & $0.75(0.03,1.45)$ \\
Hypertension & $1.00(0.06,2.81)$ & $1.03(0.08,2.80)$ & $1.46(0.08,2.88)$ \\
Proteinuria & $0.68(0.04,1.91)$ & $0.67(0.06,1.89)$ & $1.00(0.05,1.95)$ \\
HFSR & $1.42(0.07,3.86)$ & $1.41(0.05,3.88)$ & $2.05(0.09,3.98)$ \\
Elevated ALT/AST & $0.70(0.04,1.93)$ & $0.70(0.03,1.93)$ & $1.02(0.06,1.97)$ \\
Nausea/vomiting & $0.97(0.04,1.91)$ & $1.03(0.08,1.91)$ & $0.98(0.05,1.91)$ \\
Diarrhea & $0.65(0.04,1.71)$ & $0.64(0.01,1.72)$ & $0.90(0.05,1.75)$
\end{tabular}

ORR, objective response rate; DCR, disease control rate; TRAE, treatment-related adverse event; TRAE3-5, treatment-related adverse event (grade 3-5); HFSR, hand and foot syndrome. 
Table S2 The supplementary characteristics of included studies

\begin{tabular}{|c|c|c|c|c|c|c|c|c|c|c|c|c|c|c|c|c|c|c|c|}
\hline \multirow[b]{2}{*}{ Study } & \multirow[b]{2}{*}{ Year } & \multirow[b]{2}{*}{ Treatment } & \multirow{2}{*}{$\begin{array}{l}\text { Sample } \\
\text { size }\end{array}$} & \multicolumn{3}{|c|}{ ECOG } & \multicolumn{2}{|c|}{ Subtype } & \multicolumn{3}{|c|}{ EGFR } & \multicolumn{3}{|c|}{ ALK } & \multicolumn{3}{|c|}{ KRAS } & \multicolumn{2}{|c|}{ Prior oncology therapies } \\
\hline & & & & 0 & 1 & 2 & $\begin{array}{c}\text { Non- } \\
\text { squamous }\end{array}$ & Squamous & Mutated & Wild & Unknown & Mutated & Wild & Unknown & Mutated & Wild & Unknown & Chemotherapy & Targeted \\
\hline \multirow[t]{2}{*}{ Zhang } & 2012 & Apatinib & 90 & 20 & 70 & 0 & 90 & 0 & NR & NR & NR & NR & NR & NR & NR & NR & NR & NR & NR \\
\hline & & Placebo & 45 & 12 & 33 & 0 & 45 & 0 & NR & NR & NR & NR & NR & NR & NR & NR & NR & NR & NR \\
\hline \multirow[t]{2}{*}{$\operatorname{Han}(1)$} & 2018 & Anlotinib & 60 & 7 & 47 & 6 & 54 & 6 & 12 & 14 & 34 & NR & NR & NR & NR & NR & NR & 130 & NR \\
\hline & & Placebo & 57 & 3 & 49 & 5 & 50 & 7 & 9 & 11 & 37 & NR & NR & NR & NR & NR & NR & 132 & NR \\
\hline \multirow[t]{2}{*}{$\operatorname{Han}(2)$} & 2018 & Anlotinib & 294 & 59 & 233 & 2 & 228 & 53 & 93 & 201 & NR & 5 & 286 & 3 & NR & NR & NR & 294 & 158 \\
\hline & & placebo & 143 & 22 & 120 & 1 & 108 & 33 & 45 & 98 & NR & 2 & 140 & 1 & NR & NR & NR & 143 & 69 \\
\hline \multirow[t]{2}{*}{ Lu } & 2017 & Fruquintinib & 61 & 4 & 57 & 0 & 61 & 0 & 30 & 27 & 4 & NR & NR & NR & NR & NR & NR & 128 & 23 \\
\hline & & Placebo & 30 & 1 & 29 & 0 & 30 & 0 & 15 & 13 & 2 & NR & NR & NR & NR & NR & NR & 62 & 14 \\
\hline \multirow[t]{2}{*}{ Havel } & 2015 & Lenvatinib & 89 & 17 & 63 & 8 & 83 & 1 & 13 & 33 & 43 & 2 & 25 & 62 & 5 & 24 & 60 & 88 & 65 \\
\hline & & Placebo & 46 & 11 & 29 & 6 & 45 & 0 & 8 & 15 & 23 & 0 & 18 & 28 & 0 & 31 & 15 & 46 & 35 \\
\hline
\end{tabular}

NR, not reported 
A. Risk of bias graph: review authors' judgements about each risk of bias item presented as percentages across all included studies

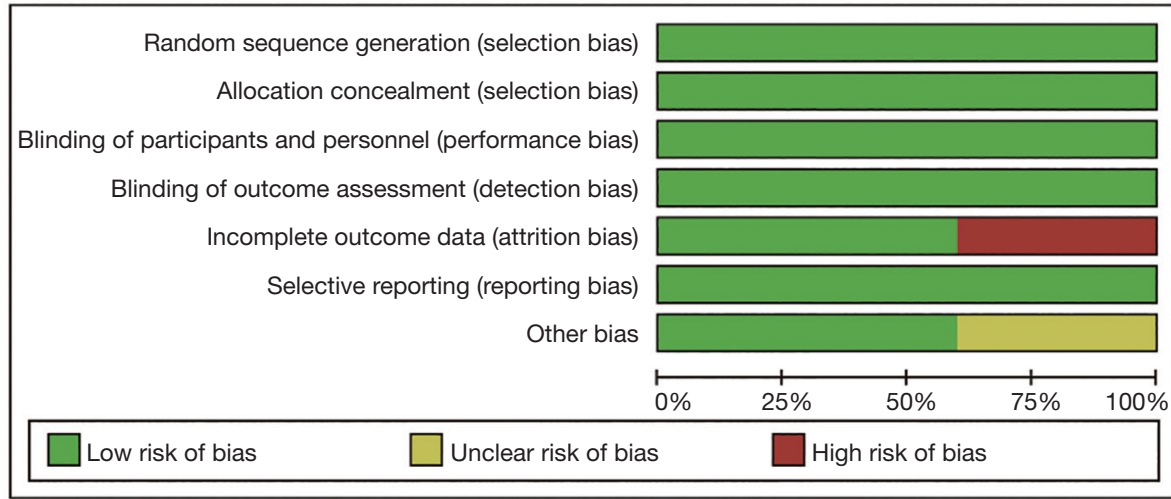

B. Risk of bias summary: review authors' judgements about each risk of bias item for each included study.

\begin{tabular}{|c|c|c|c|c|c|}
\hline 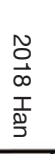 & $\begin{array}{l}\text { N } \\
\stackrel{\Xi}{J} \\
\text { ᄃ }\end{array}$ & $\begin{array}{l}\text { N } \\
\stackrel{J}{J} \\
\text { I } \\
\stackrel{I}{J}\end{array}$ & $\begin{array}{l}N \\
\text { O } \\
\text { G } \\
T \\
T \\
\mathbb{N} \\
\underline{D}\end{array}$ & 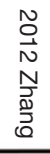 & \\
\hline+ & + & + & + & 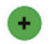 & Random sequence generation (selection bias) \\
\hline+ & + & + & + & + & Allocation concealment (selection bias) \\
\hline+ & + & + & + & + & Blinding of participants and personnel (performance bias) \\
\hline+ & + & + & + & + & Blinding of outcome assessment (detection bias) \\
\hline+ & + & + & 0 & (1) & Incomplete outcome data (attrition bias) \\
\hline+ & + & + & + & + & Selective reporting (reporting bias) \\
\hline+ & + & + & $\sim$ & $\sim$ & Other bias \\
\hline
\end{tabular}

Figure S2 Risk of bias for included studies. 


\begin{tabular}{|c|c|c|c|c|c|c|}
\hline \multicolumn{7}{|l|}{ ORR } \\
\hline Treatment & Rank1 & Rank2 & Rank3 & Rank4 & Rank5 & SUCRA \\
\hline Apatinib & 0.08 & 0.5 & 0.3 & 0.11 & 0.01 & 0.6325 \\
\hline Anlotinib & 0.04 & 0.28 & 0.37 & 0.26 & 0.05 & 0.5000 \\
\hline Fruquintinib & 0.86 & 0.07 & 0.04 & 0.02 & 0.01 & $\underline{0.9375}$ \\
\hline Lenvatinib & 0.02 & 0.15 & 0.27 & 0.43 & 0.13 & 0.3750 \\
\hline Placebo+BSC & 0 & 0 & 0.01 & 0.17 & 0.81 & 0.0475 \\
\hline \multicolumn{7}{|l|}{ DCR } \\
\hline Treatment & Rank1 & Rank2 & Rank3 & Rank4 & Rank5 & SUCRA \\
\hline Apatinib & 0.25 & 0.42 & 0.25 & 0.07 & 0.01 & 0.7075 \\
\hline Anlotinib & 0.21 & 0.25 & 0.36 & 0.14 & 0.04 & 0.6125 \\
\hline Fruquintinib & 0.49 & 0.22 & 0.18 & 0.08 & 0.02 & 0.7650 \\
\hline Lenvatinib & 0.06 & 0.11 & 0.18 & 0.56 & 0.09 & 0.3725 \\
\hline Placebo+BSC & 0 & 0 & 0.02 & 0.14 & 0.84 & 0.0450 \\
\hline \multicolumn{7}{|l|}{ PFS } \\
\hline Treatment & Rank1 & Rank2 & Rank3 & Rank4 & Rank5 & SUCRA \\
\hline Apatinib & 0.366 & 0.265 & 0.208 & 0.132 & 0.029 & 0.702 \\
\hline Anlotinib & 0.328 & 0.359 & 0.218 & 0.086 & 0.010 & $\underline{0.727}$ \\
\hline Fruquintinib & 0.203 & 0.220 & 0.283 & 0.251 & 0.044 & 0.572 \\
\hline Lenvatinib & 0.103 & 0.154 & 0.270 & 0.415 & 0.057 & 0.458 \\
\hline Placebo+BSC & 0.000 & 0.002 & 0.020 & 0.116 & 0.861 & 0.041 \\
\hline \multicolumn{7}{|l|}{ OS } \\
\hline Treatment & Rank1 & Rank2 & Rank3 & Rank4 & SUCRA & \\
\hline Anlotinib & 0.273 & 0.417 & 0.282 & 0.028 & $\underline{0.645}$ & \\
\hline Fruquintinib & 0.368 & 0.271 & 0.247 & 0.114 & 0.631 & \\
\hline Lenvatinib & 0.357 & 0.288 & 0.256 & 0.099 & 0.634 & \\
\hline Placebo+BSC & 0.001 & 0.024 & 0.215 & 0.759 & 0.089 & \\
\hline \multicolumn{7}{|l|}{ TRAE } \\
\hline Treatment & Rank1 & Rank2 & Rank3 & Rank4 & Rank5 & SUCRA \\
\hline Apatinib & 0.1 & 0.22 & 0.31 & 0.35 & 0.02 & 0.5075 \\
\hline Anlotinib & 0.22 & 0.28 & 0.27 & 0.19 & 0.04 & 0.6125 \\
\hline Fruquintinib & 0.46 & 0.2 & 0.15 & 0.14 & 0.04 & $\underline{0.7200}$ \\
\hline Lenvatinib & 0.21 & 0.3 & 0.25 & 0.2 & 0.04 & 0.6100 \\
\hline Placebo+BSC & 0 & 0 & 0.02 & 0.12 & 0.86 & 0.0400 \\
\hline \multicolumn{7}{|l|}{ TRAE3-5 } \\
\hline Treatment & Rank1 & Rank2 & Rank3 & Rank4 & SUCRA & \\
\hline Anlotinib & 0.43 & 0.39 & 0.17 & 0.02 & $\underline{0.7467}$ & \\
\hline Fruquintinib & 0.38 & 0.29 & 0.23 & 0.1 & 0.6500 & \\
\hline Lenvatinib & 0.19 & 0.29 & 0.38 & 0.13 & 0.5100 & \\
\hline Placebo+BSC & 0 & 0.03 & 0.22 & 0.75 & 0.0933 & \\
\hline Hypertension & & & & & & \\
\hline Treatment & Rank1 & Rank2 & Rank3 & Rank4 & Rank5 & SUCRA \\
\hline Apatinib & 0.26 & 0.35 & 0.27 & 0.11 & 0.01 & $\underline{0.6850}$ \\
\hline Anlotinib & 0.2 & 0.23 & 0.26 & 0.27 & 0.05 & 0.5700 \\
\hline Fruquintinib & 0.36 & 0.18 & 0.18 & 0.22 & 0.06 & 0.6400 \\
\hline Lenvatinib & 0.18 & 0.23 & 0.28 & 0.26 & 0.05 & 0.5575 \\
\hline Placebo+BSC & 0 & 0 & 0.02 & 0.14 & 0.84 & 0.0450 \\
\hline Proteinuria & & & & & & \\
\hline Treatment & Rank1 & Rank2 & Rank3 & Rank4 & Rank5 & SUCRA \\
\hline Apatinib & 0.06 & 0.21 & 0.36 & 0.31 & 0.06 & 0.4750 \\
\hline Anlotinib & 0.16 & 0.36 & 0.25 & 0.16 & 0.07 & 0.5950 \\
\hline Fruquintinib & 0.11 & 0.23 & 0.25 & 0.27 & 0.14 & 0.4750 \\
\hline Lenvatinib & 0.66 & 0.19 & 0.09 & 0.04 & 0.02 & 0.8575 \\
\hline Placebo+BSC & 0 & 0.01 & 0.05 & 0.22 & 0.72 & 0.0875 \\
\hline HFSR & & & & & & \\
\hline Treatment & Rank1 & Rank2 & Rank3 & Rank4 & Rank5 & SUCRA \\
\hline Apatinib & 0 & 0.01 & 0.31 & 0.64 & 0.04 & 0.3225 \\
\hline Anlotinib & 0 & 0.04 & 0.65 & 0.26 & 0.05 & 0.4200 \\
\hline Fruquintinib & 0.47 & 0.51 & 0.01 & 0 & 0 & 0.8575 \\
\hline Lenvatinib & 0.52 & 0.43 & 0.03 & 0.02 & 0 & 0.8625 \\
\hline Placebo+BSC & 0 & 0 & 0.01 & 0.08 & 0.91 & 0.0250 \\
\hline Elevated ALT/A & & & & & & \\
\hline Treatment & Rank1 & Rank2 & Rank3 & Rank4 & SUCRA & \\
\hline Anlotinib & 0.03 & 0.18 & 0.69 & 0.1 & 0.3800 & \\
\hline Fruquintinib & 0.63 & 0.29 & 0.06 & 0.02 & $\underline{0.8433}$ & \\
\hline Lenvatinib & 0.34 & 0.52 & 0.11 & 0.04 & 0.7233 & \\
\hline Placebo+BSC & 0 & 0.01 & 0.14 & 0.84 & 0.0533 & \\
\hline Nausea/Vomitin & & & & & & \\
\hline Treatment & Rank1 & Rank2 & Rank3 & Rank4 & Rank5 & SUCRA \\
\hline Apatinib & 0.04 & 0.11 & 0.23 & 0.31 & 0.32 & 0.3150 \\
\hline Anlotinib & 0.25 & 0.52 & 0.11 & 0.06 & 0.05 & 0.7100 \\
\hline Fruquintinib & 0.02 & 0.07 & 0.14 & 0.23 & 0.53 & 0.2000 \\
\hline Lenvatinib & 0.68 & 0.23 & 0.05 & 0.02 & 0.02 & $\underline{0.8825}$ \\
\hline Placebo+BSC & 0.01 & 0.07 & 0.47 & 0.37 & 0.08 & 0.3900 \\
\hline Diarrhoea & & & & & & \\
\hline Treatment & Rank1 & Rank2 & Rank3 & Rank4 & SUCRA & \\
\hline Anlotinib & 0.1 & 0.32 & 0.55 & 0.02 & 0.4967 & \\
\hline Fruquintinib & 0.59 & 0.23 & 0.15 & 0.03 & $\underline{0.7933}$ & \\
\hline Lenvatinib & 0.31 & 0.44 & 0.22 & 0.03 & 0.6767 & \\
\hline Placebo+BSC & 0 & 0.01 & 0.08 & 0.91 & 0.0333 & \\
\hline
\end{tabular}

Figure S3 Rank probabilities with SUCRA value for different outcomes in four kinds of multiple-target TKIs. TKI, tyrosine kinase inhibitor; ORR, objective response rate; DCR, disease control rate; PFS, progression-free survival; OS, overall survival; TRAE, treatment-related adverse event; TRAE3-5, treatment-related adverse event (grade 3-5); HFSR, hand and foot syndrome; BSC, best support care; SUCRA, surface under the cumulative ranking curve. 